\title{
Compósitos $\mathrm{SiC}_{\mathrm{f}} / \mathrm{SiC}$ utilizados em sistemas de proteção térmica
}

\author{
( $\mathrm{SiC}_{f} / \mathrm{SiC}$ composites for thermal protection systems)
}

\author{
M. Florian', L. E. Carvalho', K. Iha', M. L.A. Graça', C. A. A. Cairo ${ }^{2}$ \\ ${ }^{I}$ Depto. de Engenharia Aeronáutica e Mecânica - ITA - CTA \\ Pça. Mal. Eduardo Gomes 50, S. José dos Campos, SP 12228-901 \\ ${ }^{2}$ Divisão de Materiais - Instituto de Aeronáutica e Espaço - CTA \\ Pça Mal. Eduardo Gomes, 50, S. José dos Campos, SP 12228-904
}

\begin{abstract}
Resumo
Compósitos de carbeto de silício $(\mathrm{SiC})$ reforçado com fibras de carbeto de silício $\left(\mathrm{SiC}_{\mathrm{f}}\right)$ são materiais candidatos em potencial para utilização em sistemas de proteção térmica em altas temperaturas devido principalmente à boa condutividade térmica na direção da fibra e muito baixa condutividade térmica na direção transversal à fibra, alta dureza, estabilidade térmica e à corrosão por oxidação. O compósito $\mathrm{SiC}_{\mathrm{f}} \mathrm{SiC}$ possui uma matriz de $\mathrm{SiC}$ reforçada com fibras contínuas policristalinas de $\mathrm{SiC}$ e é obtido por reações de conversão em altas temperaturas e atmosfera controlada, utilizando o compósito carbono/carbono como precursor. O processo de Reação Química em Vapor (CVR) foi utilizado para a fabricação de compósitos $\mathrm{SiC}_{\mathrm{f}} / \mathrm{SiC}$ com alta pureza na fase de $\mathrm{SiC}-\beta$. O compósito precursor de carbono/carbono foi fabricado com fibra de carbono não estabilizada e matriz carbonosa derivada da resina fenólica na forma de carbono isotrópico. O compósito convertido exibiu uma densidade de 1,75 g/cm³, com $40 \%$ de porosidade aberta e resistência à flexão de $80 \mathrm{MPa}$ medida por ensaio flexão em 4 pontos. A área especifica medida pela técnica de BET é dependente da temperatura de conversão e das condições inicias do precursor de carbono, podendo chegar a $18 \mathrm{~m}^{2} / \mathrm{g}$.
\end{abstract}

Palavras-chave: compósito C/C, compósito $\mathrm{SiC}_{f} / \mathrm{SiC}$, caracterização, microestrutura, reação química em vapor (CVR).

\begin{abstract}
Composites based on silicon carbide are potential candidate materials for thermal protection systems mainly due to its good thermal conductivity in fiber direction and very low transversal thermal conductivity, high hardness, corrosion and thermal resistance. $\mathrm{SiC}$ SiC composite presents a SiC matrix reinforced with SiC polycrystalline continuous fibers. The composite was obtained by conversion reactions at high temperature and controlled atmosphere from a carbon/carbon composite precursor. The CVR process was used to fabricate SiC/SiC composite with crystalline high-purity $\beta$-SiC from a carbon-carbon precursor fabricated with nonstabilized carbon fiber and carbon matrix derived from phenolic resin. The converted composite exhibited a bulk density of 1.75 $\mathrm{g} / \mathrm{cm}^{3}, 40 \%$ open porosity and flexural strength of $80 \mathrm{MPa}$ measured by four-point bending testing. The total specific area of $18 \mathrm{~m}^{2} / \mathrm{g}$ was evaluated by BET technique.
\end{abstract}

Keywords: C/C composite, $\mathrm{SiC}_{\mathrm{f}} / \mathrm{SiC}$ composite, characterization, microstructure, chemical vapor reaction (CVR).

\section{INTRODUÇÃO}

O carbeto de silício (SiC) tem sido extensivamente utilizado em várias aplicações, inclusive na área aeroespacial, como material estrutural por causa de suas características como excelente propriedades mecânicas em alta temperatura, estabilidade térmica e química [1-3] e resistência à oxidação em atmosferas ricas em oxigênio e alta emissividade de calor $(\sim 0,95)$ Uma possibilidade de aplicação na área espacial está na composição de sistemas de proteção térmica de veículos orbitais recuperáveis. O carbeto de silício disposto na forma de um compósito bidirecional reforçado com fibras de SiC- $\beta$, possui alta condutividade térmica na direção das fibras $(70 \mathrm{~W} / \mathrm{m} . \mathrm{K})$ e uma baixa condutividade na direção transversal ao plano das fibras (15 W/m.K) [4].

Todos os sistemas de proteção térmica agem de forma a evitar dano causado pelo calor na estrutura, nos sensores e carga útil do veiculo espacial. No caso do sistema que utiliza $\mathrm{SiC}_{\mathrm{f}} / \mathrm{SiC}$ o calor é dissipado pelo mecanismo conhecido como "resfriamento por radiação". Nesse caso o fluxo de calor é refletido de volta para o espaço pela alta emissividade de calor da camada externa do compósito $\mathrm{SiC}_{\mathrm{f}} / \mathrm{SiC}$. Esse mecanismo é muito efetivo em órbita, uma vez que a taxa de transferência de calor é proporcional à emissividade e a diferença entre a temperatura do veículo e do espaço elevada à quarta potência [5]. Ainda, a alta condutividade térmica do $\mathrm{SiC}_{\mathrm{f}} / \mathrm{SiC}$ no plano das fibras, é responsável pela distribuição do calor por toda a calota da proteção, evitando a dano maior pela concentração de calor no ponto de estagnação e a baixa condutividade na direção transversal evita o aquecimento da parte interna do veiculo.

As técnicas de processamento do compósito $\mathrm{SiC}_{\mathrm{f}} / \mathrm{SiC}$ como deposição por vapor químico (CVD) [6,7], infiltração 
química à vapor (CVI) [8, 9], impregnação de polímeros e pirólise (PIP) [10, 11], reação por sinterização (RS) [11], utilizam como pré-forma fibras de $\mathrm{SiC}$ do tipo Tyranno e Nicalon. Além do alto custo inicial das fibras, o processo de impregnação da matriz é demorado e caro. Neste trabalho são apresentados os resultados da obtenção de $\mathrm{SiC}_{\mathrm{f}} / \mathrm{SiC}$ pela técnica de CVR [12, 13]. A obtenção do compósito $\mathrm{SiC}_{\mathrm{f}} / \mathrm{SiC}$ pela técnica de CVR consiste na transformação do compósito carbono/carbono precursor em SiC, por meio de reações químicas que ocorrem entre o gás SiO, que é gerado por reações entre os componentes de pós-metálico e cerâmico, e o carbono do compósito precursor, por meio de reação sólido-gás [14]. A extensão da reação depende da temperatura e da quantidade de gás $\mathrm{SiO}$ gerado. A mistura $\mathrm{Si}$ $+\mathrm{SiO}_{2}$ gera vapor de $\mathrm{SiO}$ como mostrado na equação A:

$$
\mathrm{Si}_{(\mathrm{s})}+\mathrm{SiO}_{2(\mathrm{~s})} \rightarrow 2 \mathrm{SiO}_{(\mathrm{g})}
$$

a pressão de vapor no equilíbrio é de 100 Pa ou maior em temperaturas acima de $1270^{\circ} \mathrm{C}$ [14]. O gás de $\mathrm{SiO}$ formado reage com o carbono segundo a equação B:

$$
2 \mathrm{SiO}_{(\mathrm{g})}+2 \mathrm{C}_{(\mathrm{s})} \rightarrow \mathrm{SiC}_{(\mathrm{s})}+\mathrm{CO}_{(\mathrm{g})}
$$

A mistura utilizada na geração é composta por $\mathrm{SiO}_{2}$ e Si metálico [12]. O gás de SiO que é altamente reativo, penetra no compósito C-C transformando tanto a matriz como as fibras em $\mathrm{SiC}$ altamente puro na fase de $\mathrm{SiC}-\beta$.

A obtenção do compósito $\mathrm{SiC}_{\mathrm{f}} / \mathrm{SiC}$ está inserido no projeto de obtenção de materiais isolantes térmicos para utilização de veículos orbitais recuperáveis (Projeto SARA), desenvolvido no IAE-CTA.

\section{MATERIAIS E MÉTODOS}

\section{Matérias-primas}

Os materiais utilizados na forma de pó ou como a mistura do pó cementante, no processo de conversão do compósito C/ $\mathrm{C}$ em $\mathrm{SiC}_{\mathrm{f}} / \mathrm{SiC}$ foram: Si da Elektroschmeltzwerk Kempten GMBH, pureza 99,9\% e tamanho médio de partícula 10 $\mu \mathrm{m} ; \mathrm{SiO}_{2}$ produzido pela Mineração Jundu, pureza $99 \%$ e tamanho médio de partícula $<2 \mu \mathrm{m}$; fibra de carbono twill, T-10 EKHO, derivado do precursor poliacrilonitrila, carbonizada; resina fenólica Resafen 8121, produzida pela Reichhold-Resana Ind. Quim S/A, utilizada como precursor da matriz de carbono.

\section{Mistura dos pós cerâmicos}

A mistura de pó $50 \% \mathrm{SiO}_{2}+50 \% \mathrm{Si}$ (\%massa) foi realizada a seco em moinho planetário por 30 min e passado em peneira de 80 mesh para desaglomeração. Tanto a mistura cerâmica como os corpos de prova de C/C foram mantidos em estufa à $150{ }^{\circ} \mathrm{C}$ por $12 \mathrm{~h}$ para secagem.

\section{Fabricação do compósito $C / C$}

O compósito C/C foi preparado impregnando-se o tecido de carbono com a resina fenólica até completar a seqüência de 8 camadas. Em seguida, o laminado foi colocado em autoclave até a temperatura de $130{ }^{\circ} \mathrm{C}$ com taxa de aquecimento de $5^{\circ} \mathrm{C} / \mathrm{min}$ e pressão de $0,3 \mathrm{MPa}$ para a cura da resina. $\mathrm{O}$ compósito foi carbonizado à $1000^{\circ} \mathrm{C}$ em atmosfera de argônio, a uma razão de aquecimento de $20^{\circ} \mathrm{C} / \mathrm{h}$.

\section{Transformação do compósito $\mathrm{C} / \mathrm{C}$ em $\mathrm{SiC} / \mathrm{SiC}$}

A mistura cementante $\mathrm{SiO}_{2} / \mathrm{Si}$ foi colocada em um cadinho de grafite e compactada manualmente. O compósito C/C a ser transformado foi colocado próximo da mistura de pós como é mostrado na Fig. 1.

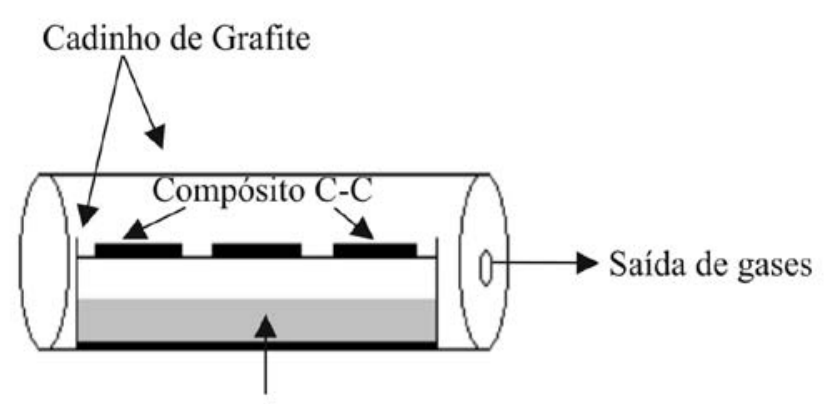

Mistura do pó cerâmico

Figura 1: Desenho esquemático da transformação do C/C em SiC/SiC. [Figure 1: Schematic representation of the transformation of $\mathrm{C} / \mathrm{C}$ composite in SiC/SiC composite.]

Após a compactação do pó, o cadinho foi colocado em estufa para secagem à $200^{\circ} \mathrm{C}$ por $12 \mathrm{~h}$. O tratamento térmico foi realizado em forno resistivo em vácuo. A $1400^{\circ} \mathrm{C} / 3 \mathrm{~h}$ com taxa de aquecimento de $10^{\circ} \mathrm{C} / \mathrm{min}$. Terminado o tratamento térmico, o compósito transformado foi limpo em ultra-som com acetona.

\section{Caracterização do compósito}

A microestrutura do compósito $\mathrm{SiC}_{\mathrm{f}} / \mathrm{SiC}$, as observações da extensão de transformação, a interface entre a fibra e matriz foram avaliadas por microscopia eletrônica de varredura no equipamento LEO modelo $435 \mathrm{Vpi}$ com EDS da Oxford modelo 7059 acoplado. A análise química do compósito transformado foi realizada por EDS (Energia Dispersiva de raios X). A identificação das fases presentes no compósito $\mathrm{SiC}_{\mathrm{f}} / \mathrm{SiC}$ foi realizada pelo método de difração de raios X, no aparelho Philips modelo X'pert PW 1830, utilizando radiação $\mathrm{Cu}-\mathrm{K} \alpha$ e varrendo as amostras de 0 a $80^{\circ}$ com velocidade de $1 \%$ min. O método utilizado para a determinação da massa especifica e porosidade aberta do compósito foi o de Arquimedes [15]. A resistência à flexão 
foi determinada pelo ensaio de teste de flexão em 4 pontos com velocidade de $0,5 \mathrm{~mm} / \mathrm{min}$ e razão vão/espessura de 15, utilizando máquina Instron 4301 com célula de carga $500 \mathrm{kN}$. A análise de área superficial (BET) foi determinada utilizando equipamento ASAP 2010 V5.02.

\section{RESULTADOS E DISCUSSÃO}

A Fig. 2 mostra uma vista geral do compósito C-C antes da conversão, pode-se notar os feixes de fibras de carbono nas direções longitudinal e transversal e os vazios deixados após a carbonização do precursor da matriz carbonosa. Os vazios maiores estão localizados nos espaços entre os feixes de fibras e os vazios menores entre as fibras do mesmo feixe. A Fig. 3 mostra as fibras do compósito após a conversão em SiC- $\beta$. Observa-se que os aspectos microestruturais são mantidos após o tratamento térmico de conversão.

Pela diferença de coloração das microfotografias obtidas

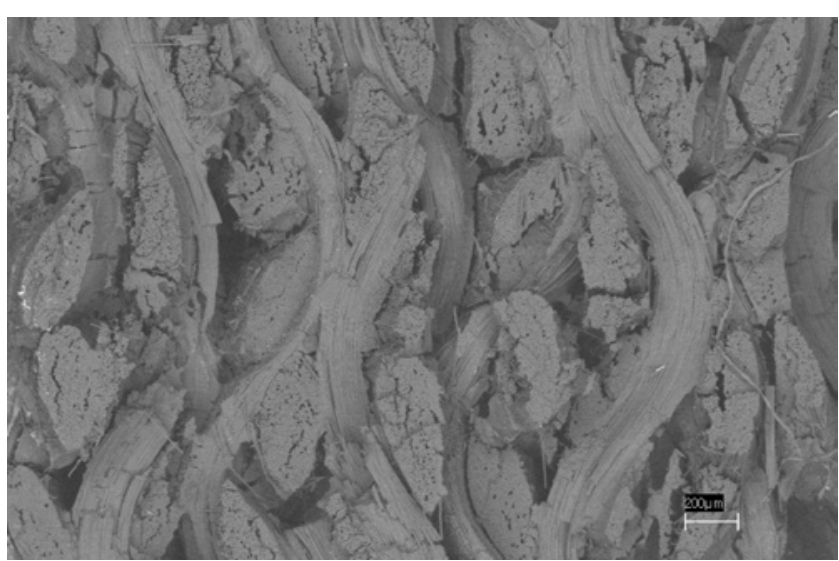

Figura 2: Micrografia da visão geral do compósito $\mathrm{C} / \mathrm{C}$ após carbonização, obtida por MEV, no modo retroespalhado (BSE).

[Figure 2: General view by SEM/BSE of C/C composite after carbonization.]

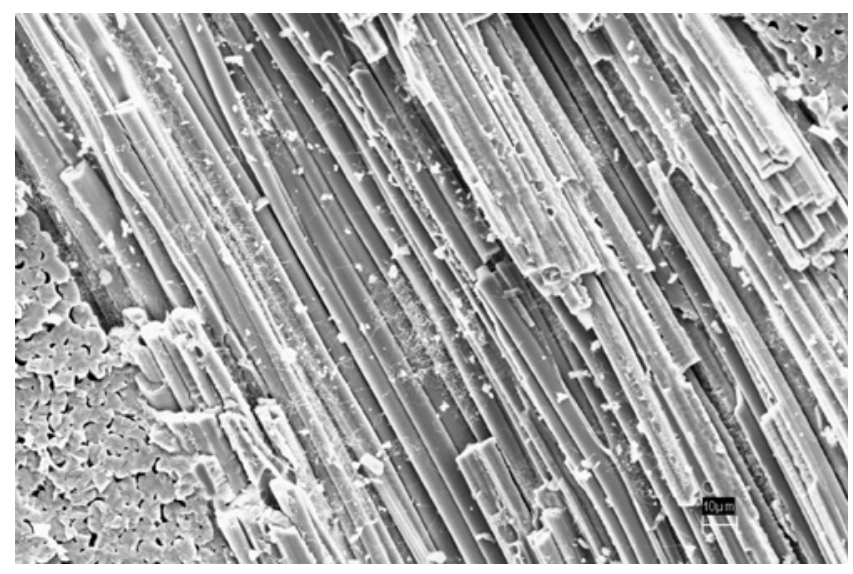

Figura 3: Micrografia do compósito transformado $\mathrm{SiC}_{\mathrm{f}} / \mathrm{SiC}$ pela mistura $50 \% \mathrm{SiO}_{2}+50 \% \mathrm{Si}$ tratado a $1400{ }^{\circ} \mathrm{C} / 3 \mathrm{~h}$, obtida por $\mathrm{MEV}$, no modo retroespalhado (BSE).

[Figure 3: SEM/BSE micrographs of SiC/SiC composite transformed by the mixture of $50 \% \mathrm{SiO}_{2}+50 \%$ Si treated at $1400{ }^{\circ} \mathrm{C} / 3 \mathrm{~h}$.] no MEV no modo de elétrons retroespalhados, pode-se observar a extensão da reação (Fig. 3). O carbono se apresenta com a coloração cinza escura enquanto o SiC de coloração clara brilhante, devido as diferenças de peso atômico.

Na Fig. 4 é exibido o detalhe do compósito transformado, mostrando as fibras de $\mathrm{SiC}$ inseridas na matriz de $\mathrm{SiC}$, já na Fig. 5 observa-se somente a fibra policristalina na fase SiC- $\beta$. $\mathrm{Na}$ análise de elementos por energia dispersiva de raios $\mathrm{X}$, o compósito transformado apresenta somente os picos referentes aos elementos Si e C, como mostra a Fig. 6, com o elemento Si presente na totalidade da área analisada.

A observação feita por MEV é confirmada pelos difratogramas de raios X, Figs. 7 e 8.

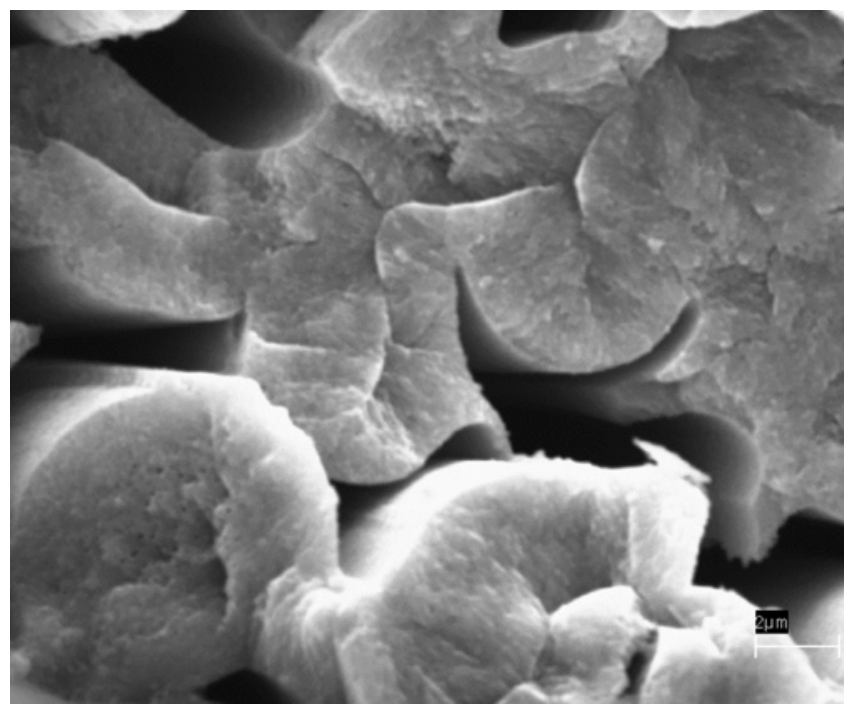

Figura 4: Detalhe do compósito $\mathrm{SiC}_{\mathrm{f}} / \mathrm{SiC}$ exibindo a fibra e a matriz de SiC.

[Figure 4: Detail of SiC/SiC exhibiting both SiC fiber and matrix.]

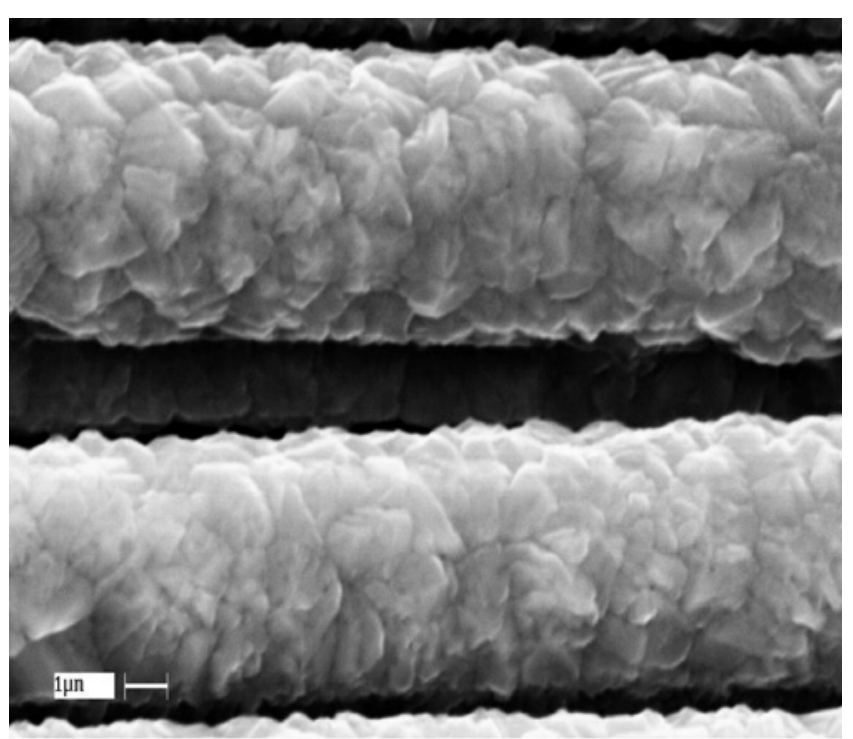

Figura 5: Fibra cristalina de SiC- $\beta$.

[Figure 5: $\beta$-SiC crystalline fiber.] 


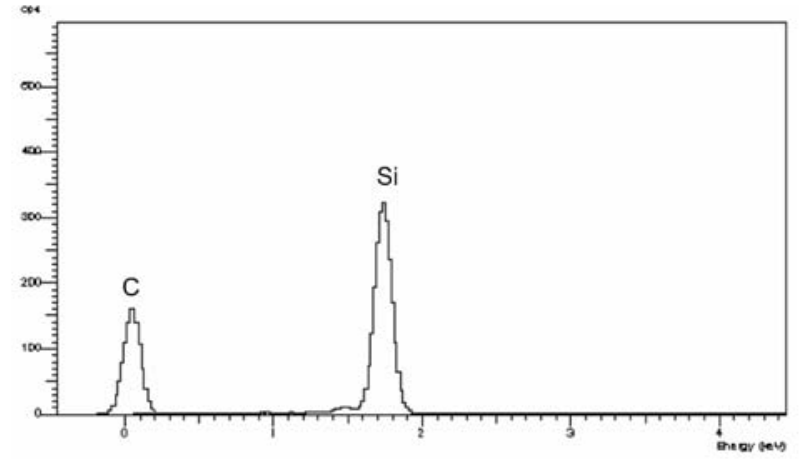

Figura 6: Análise do compósito transformado, mostrando a presença dos elementos Si e C.

[Figure 6: Analysis of transformed composite showing Si and C.]

Pelo difratograma da Fig. 7, é possível observar somente a fase grafite, com pico de intensidade $100 \%$ em $2 \theta$ igual a 26,5. Após tratamento térmico de conversão, não é observado a presença de nenhum pico de carbono, somente a presença da fase SiC- $\beta$ (Fig. 8).

A massa especifica aparente do compósito $\mathrm{SiC}_{\mathrm{f}} / \mathrm{SiC}$, medida pelo método de Arquimedes, foi de $1,75 \mathrm{~g} / \mathrm{cm}^{3} \mathrm{com}$ porosidade aparente total de $40 \%$. Como o compósito $\mathrm{SiC}_{\mathrm{f}}{ }^{\prime}$ $\mathrm{SiC}$ foi obtido pela conversão do $\mathrm{C} / \mathrm{C}$, a porosidade aberta é igual a do precursor. Compósitos mais densos podem ser obtidos pela densificação do $\mathrm{C} / \mathrm{C}$ antes da conversão. A porosidade medida está associada aos vazios entre os feixes de fibras indicadas na Fig. 2 e os vazios entre as fibras dentro de um mesmo feixe como pode ser visto na Fig. 4.

$\mathrm{O}$ valor de ruptura em flexão foi de $80 \mathrm{MPa}$. Os valores de massa específica aparente, porosidade aparente e resistência à flexão são comparados com aqueles materiais obtidos por outros métodos na Tabela I.

Considerando-se que o processo de obtenção do $\mathrm{SiC}_{\mathrm{f}} \mathrm{f}$ SiC é o da conversão do carbono em carbeto de silício por reação sólido-gás em altas temperaturas, o compósito obtido possui um arranjo microestrutural semelhante ao do precursor. A alta porosidade apresentada pelo $\mathrm{SiC}_{\mathrm{f}} / \mathrm{SiC}$, obtido neste trabalho quando comparada com materiais da Tabela I pode ser trabalhada na etapa de impregnação das fibras de carbono durante a fabricação do compósito

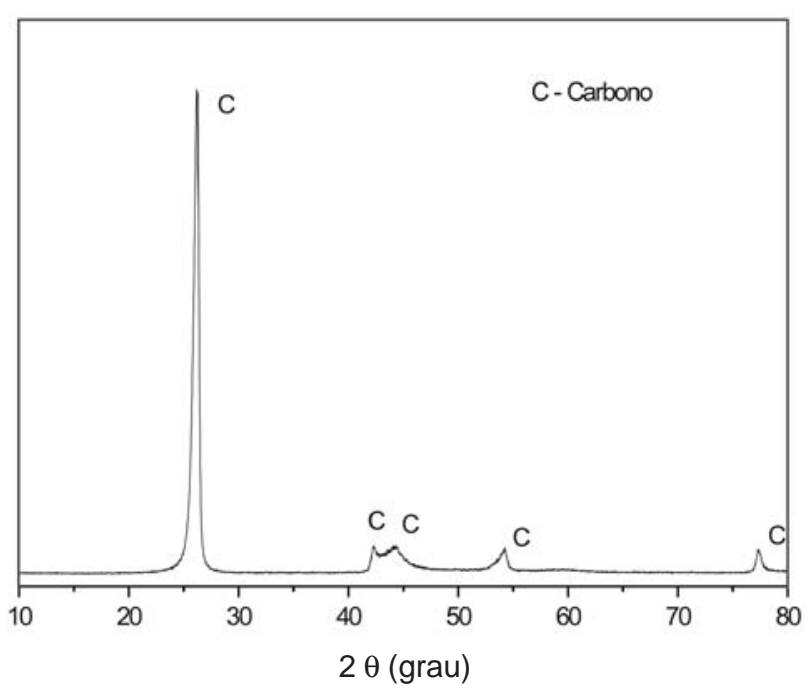

Figura 7: Difratograma de raios $X$ do compósito $C / C$ precursor carbonizado.

[Figure 7: X-ray diffraction pattern of carbonized C/C precursor composite.]

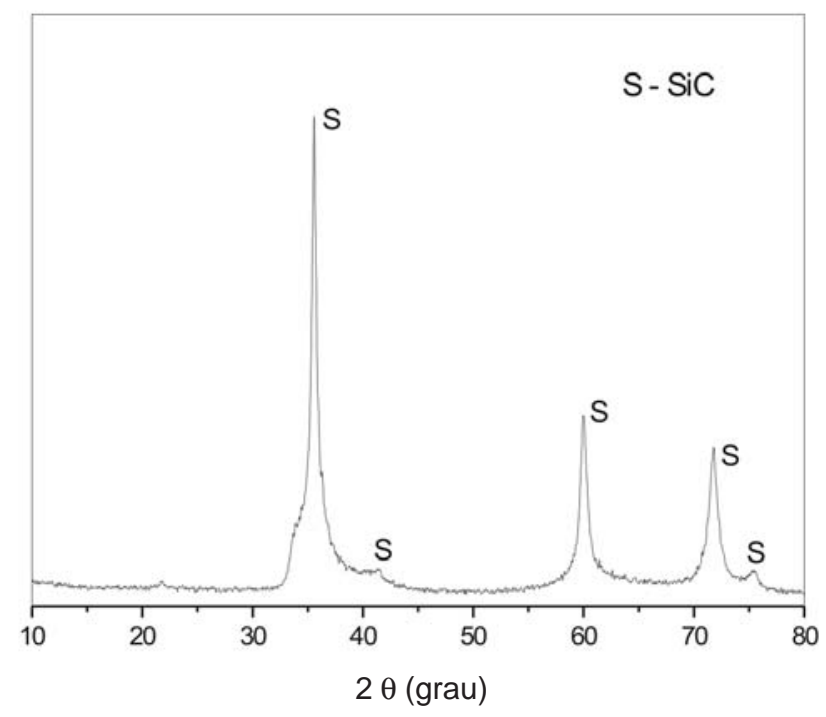

Figura 8: Difratograma de raios $\mathrm{X}$ do compósito transformado obtido pela mistura $50 \% \mathrm{SiO}_{2}+50 \%$ Si tratado à $1600{ }^{\circ} \mathrm{C} / 3 \mathrm{~h}$.

[Figure 8: X-Ray diffraction pattern of transformed composite obtained by the mixture of $50 \% \mathrm{SiO}_{2}+50 \%$ Si treated at $1600^{\circ} \mathrm{C} / 3 \mathrm{~h}$.]

Tabela I - Comparação dos valores de massa especifica, porosidade aberta e resistência à flexão para o compósito $\mathrm{SiC}_{\mathrm{f}} / \mathrm{SiC}$. [Table I - Values of specific weight, open porosity and four point bending stress for SiC/SiC composite.]

\begin{tabular}{lccc}
\hline & $\begin{array}{c}\text { CVR Reação } \\
\text { Química via Vapor }\end{array}$ & $\begin{array}{c}\text { PIP }^{[5]} \text { Infiltração } \\
\text { e Pirólise de Polímero }\end{array}$ & $\begin{array}{c}\text { CVI }^{[3]} \text { Infiltração } \\
\text { Química via Vapor }\end{array}$ \\
\hline Massa Especifica (g/cm $\left.{ }^{3}\right)$ & 1,75 & 2,66 & $2,38-3,07$ \\
Porosidade Aberta (\%) & 40 & $0,8-1$ & $10-20$ \\
Resistência à Flexão (MPa) & 80 & $230-110$ & $140-260$ \\
Resistência Específica & 46 & $41-86$ & $46-109$ \\
\hline
\end{tabular}


precursor. O numero de impregnações sucessivas leva a uma maior densificação e preenchimento da porosidade advinda da contração da resina durante a etapa de carbonização para a obtenção da matriz carbonosa.

No entanto, para a aplicação como proteção térmica de veículos aeroespaciais, o peso é um fator relevante. A resistência especifica, que é a razão entre a resistência mecânica pela massa específica do material, é uma medida essencial para a avaliação do potencial de uso dos materiais. Considerando-se esse parâmetro, o $\mathrm{SiC}_{\mathrm{f}} / \mathrm{SiC}$, obtido por reações de conversão apresenta uma relação custo-benefício favorável frente aos materiais obtidos por outros métodos.

Considerando-se também que a porosidade, principalmente aquela relacionada aos vazios entre as fibras podem alterar as propriedades térmicas do material, atuando principalmente na diminuição da condutividade térmica na direção transversal aos planos das fibras, pode-se esperar um ganho em termos das propriedades de isolamento da camada protetora, enquanto que a condutividade na direção das fibras ao longo de todo o material, não é alterada pela porosidade.

A área específica total, aproximadamente 18,50 $\mathrm{m}^{2} /$ g, qualifica o material para uso em aplicações onde esta característica pode ser explorada, associada à estabilidade química e mecânica do compósito em altas temperaturas.

\section{CONCLUSÕES}

O compósito $\mathrm{SiC}_{\mathrm{f}} / \mathrm{SiC}$ pode ser obtido pela conversão de compósito $\mathrm{C} / \mathrm{C}$, por meio das reações químicas em altas temperaturas, envolvendo o gás reagente $\mathrm{SiO}$ como produto das reações entre $\mathrm{SiO}_{2(\mathrm{~s})}$ e Si metálico.

A microestrutura do compósito obtido é uma réplica do precursor de carbono. A transformação ocorre para produzir fibras policristalinas pura e matriz de SiC- $\beta$.

A resistência específica do compósito obtido, considerandose o nível de porosidade em torno de $40 \%$, que está relacionada em grande parte aos vazios entre as fibras individuais, com tamanhos de aproximadamente $1 \mu \mathrm{m}$ e a forma alongada destes vazios que acompanham toda a extensão das fibras permitem avaliar o compósito como um potencial candidato para o uso em sistemas de proteção térmica para veículos de reentrada, onde são requeridas propriedades como alta resistência especifica, baixo peso, e baixa condutividade térmica na direção transversal às fibras.

\section{AGRADECIMENTOS}

Os autores agradecem ao CNPq e a FAPESP (03/09435-8).

\section{REFERÊNCIAS}

[1] M. Kotani, T. Inoue, A. Koyama, Y. Kaoh, K. Okamura, Mater. Sci. Eng. A357 (2003) 376.

[2] J. Schulte-Fischedick, A. Zern, J. Mayer; M. Rühle, M. Frie $\beta$, W. Krenkel, R. Kochendörfer, Mater. Sci. Eng. A332 (2002) 146.

[3] T. Yano, K. Budiyanto, K. Yoshida, T. Iseki, Fusion Eng. and Design 41 (1998) 157.

[4] W. Kowbel, C.A. Bruce, K.L. Tsou, K. Patel, J.C. Withers, G. E. Youngblood, J. Nuclear Mater. 283-287 (2000) 570.

[5] J. D. Guthrie, B. Battat, B. K. Severin, Materials Ease AMPTIAC 11 (2000).

[6] J. S. Lee, T. Yano, J. Eur. Ceram. Soc. 24 (2004) 25.

[7] L. Cheng, Y. Xu, L. Zhang, X. Yin, Carbon 37 (1999) 977.

[8] H. Araki, N. Tetsuji, W. Yang, A. Hohyama, J. Nuclear Mater. 307-311 (2002) 1210.

[9] Y. G. Roman, D. P. Stinton, Mater. Res. Soc. Symp. Proc. (1995) 343.

[10] M. Kotani, A. Kohyama, Y. Katoh, J. Nuclear Mater. 289 (2001) 37.

[11] M. Kotani, T. Inoue, A. Kohyama, Y. Katoh, K. Okamura, Mater. Sci.Eng. A357 (2003) 376.

[12] S. Ohsaki, D. H. Cho, H. Sano, Y. Uchiyama, K. Kobayashi, Key Eng. Mater. 159-160 (1999) 89.

[13] M. Florian, Diss. Mestrado, ITA (2002).

[14] U. Setiowati, S. Kimura, J. Am. Ceram. Soc. 80 (1997). 757.

[15] Standard Test Methods for Apparent Porosity, Water Absorption, Apparent Specific Gravity and Bulk Density of Burned Refractory Brick and Shapes by Boiling Water. 